\title{
Estimating Survival in Patients with Non-Small-Cell Lung Cancer and Brain Metastases: A Verification of the Graded Prognostic Assessment for Lung Cancer Using Molecular Markers (Lung-molGPA)
}

This article was published in the following Dove Press journal: OncoTargets and Therapy

$\mathrm{Ji} \mathrm{Li}{ }^{1, *}$

Wang Jing ${ }^{2, *}$

Xiaoyang Zhai ${ }^{2}$

Wenxiao Jia $^{2}$

Hui Zhu ${ }^{2}$

Jinming $\mathrm{Yu}^{\mathrm{I}}$

'Department of Oncology, Renmin Hospital of Wuhan University, Wuhan, Hubei, 430060, People's Republic of China; ${ }^{2}$ Department of Radiation Oncology, Shandong Cancer Hospital and Institute, Shandong First Medical University and Shandong Academy of Medical Sciences, Jinan, 250II7, Shandong, People's Republic of China

*These authors contributed equally to this work
Correspondence: Hui Zhu; Jinming Yu Tel +86-53I-67626782;

$+86-531-67626947$

Email drzhuh@।26.com;

sdyujinming@।63.com
Purpose: A new tool based on clinical characteristics and molecular factors (LungmolGPA) was developed to predict the survival of patients with non-small-cell lung cancer but was has not been validated. This study aims to validate the feasibility of the LungmolGPA in NSCLC.

Patients and Methods: Patients diagnosed NSCLC between Feb 2012 and July 2018 were retrospectively reviewed and scored using the Lung-molGPA tool to compare clinical outcomes. Hazard ratios (HRs) and 95\% confidence intervals (CIs) were calculated by Cox regression analyses.

Results: A total of 618 patients (524 adenocarcinoma [ADC], 94 non-adenocarcinoma [nonADC]) were collected. For all patients, the median survival time (MST) was 33.0 months (33.6 and 28 months in the ADC and non-ADC groups, respectively; $p=0.21$ ). In the ADC group, the MST for patients with a Lung-molGPA score of 3.5 to 4 was more than 4 years, while the MST was only 25 months in patients scoring $0-1,30.0$ months in patients scoring $1.5-2$, and 35.0 months for scores of 2.5-3 ( $p=0.048)$. For the non-ADC group, the MST for scores $0-1,1.5-2,2.5-3$, and 3.5-4 were $12.0,20.2,29.0$, and 33.0 months, respectively $(p=0.017)$.

Conclusion: Our findings provided evidence validating the Lung-molGPA score as a useful tool to determine treatment strategies and to predict prognosis. The model is still exploratory and needs to be evaluated further in combination with additional prognostic markers.

Keywords: NSCLC, GPA, brain metastasis, survival, prognosis

\section{Introduction}

Lung cancer is the first leading cause of cancer-related deaths in China and worldwide. $^{1,2}$ Brain metastases (BM) are frequently observed in non-small-cell lung cancer (NSCLC), which undoubtedly exerts a devastating influence on the survival and quality of life of patients. About $40 \%$ of patients present BM during the course of the disease. $^{3,4}$ The median overall survival (OS) was reported to be merely 4-6 months after whole brain radiation therapy (WBRT) in the chemotherapy era. ${ }^{3,4}$

With the recent advent of molecularly targeted therapies, it is evident that NSCLC patients with BM are an extremely heterogeneous population. Identifying various subgroups with different prognosis is essential to precisely individualize treatment and especially to design clinical trials. Several models have 
focused on predicting survival for patients with BM such as Recursive Partitioning Analysis (RPA), which was established by the Radiation Therapy Oncology Group (RTOG) 20 years ago, based on the data from 1200 patients in 3 clinical trials. ${ }^{5}$ In this model, patients are stratified according to age, the Karnofsky Performance Status (KPS), control of primary tumor, and extracranial metastases, into three classes of disease with median survival ranging from 2.3 to 7.1 months. Furthermore, the Diagnosis Specific Graded Prognostic Assessment (DS-GPA) score was established in 2012 and takes into consideration age, and four clinical factors: KPS, extracranial metastases, and the number of brain metastases. ${ }^{6}$ Median survival ranges from 3.0 to 14.8 months according to the four classes of disease.

It is known that advanced NSCLC patients harboring epidermal growth factor receptor $(E G F R)$ and anaplastic lymphoma kinase $(A L K)$ alterations treated with tyrosine kinase inhibitors (TKIs), achieve an OS superior to that of patients without any driving gene mutations. However, NSCLC patients with EGFR mutations experience a higher incidence of $\mathrm{BM}$, ranging from $44 \%$ to $63 \%$. Thus, to predict the survival of NSCLC with BM, molecular factors should also be considered. Sperduto et al updated the graded prognostic assessment for Lung Cancer Using Molecular Markers (Lung-molGPA) based on the clinical characteristics of 2186 patients to include age, KPS, extracranial metastases, number of $\mathrm{BM}$, and $E G F R$ and $A L K$ alterations in patients with ADC. ${ }^{7}$ The median OS for the entire cohort was 12 months, and patients harboring EGFR and $A L K$ alterations and with Lung-molGPA scores of 3.5 to 4.0 had a median survival of nearly 4 years.

Nonetheless, published studies providing evidence for the validation of the Lung-molGPA based on large patient samples are lacking. Hence, we conducted the present study and reviewed the records of 618 patients, with the aim to validate the clinical utility of the Lung-molGPA scoring system.

\section{Patients and Methods}

\section{Patients}

The records of patients diagnosed with NSCLC treated for BM between February 2012 and June 2018 at our cancer center were reviewed. All patients were staged according to the criteria of AJCC 7th edition. At the initial diagnosis, whole-body systemic evaluation was performed to evaluate the disease stage, including history and physical examination, blood profile, cervical ultrasound or computed tomography
(CT), chest and abdomen enhanced contrast CT, brain enhanced contrast CT or magical resonance imaging, positron emission tomography-CT was not routinely performed in our cancer center and data was available only for a few patients.

\section{Graded Prognostic Assessment}

All patients were stratified based on the Lung-molGPA score. The Lung-molGPA score was calculated according to the graded prognostic assessment (GPA) index using a web-based interface (http://brainmetgpa.com/), and the supplementary calculations were carried out according to those reported in previous studies. ${ }^{7}$ The type of treatment was not considered because the purpose of the LungmolGPA was score is to estimate survival prior to treatment. In the Lung-molGPA scoring system, according to the size of the effect on the survival, KPS from 90 to 100 , no extracranial metastases, $>4 \mathrm{BM}$ and $E G F R$ or $A L K$ positivity were given a score of 1 , age $<70$ years and $1-4 \mathrm{BM}$ were given a score of 0.5. Thus, the maximum score remained 4.0. The parameters of the new Lung-molGPA are detailed in Table 1. All enrolled patients were divided into four groups based on the final scores: $0-1,1.5-2,2.5-3,-4$ (Table 1 ).

\section{Statistical Analysis}

OS was defined from the date of initial treatment of BM to the date of death due to any cause, or to the latest follow-up. Progression-free survival (PFS) was defined as the time from the date of initial treatment of BM to the date of disease progression, or death due to any cause. OS and PFS were calculated by the Kaplan-Meier method, and differences in survival curves between the groups were evaluated by the Log rank test. Two-sided $p$ values $<0.05$ were considered

Table I Lung-molGPA Scoring Chart for NSCLC

\begin{tabular}{|l|l|l|l|}
\hline \multirow{2}{*}{ Parameter } & \multicolumn{3}{|c|}{ GPA Scoring Criteria } \\
\cline { 2 - 4 } & 0 & 0.5 & I \\
\hline Age, yrs & $\geq 70$ & $<70$ & NA \\
\hline KPS & $<80$ & 80 & $90-100$ \\
\hline ECM & Present & & Absent \\
\hline BM & $>4$ & $1-4$ & NA \\
\hline Gene Status & $\begin{array}{l}\text { EGFR neg/unk and } \\
\text { ALK neg/unk }\end{array}$ & NA & $\begin{array}{l}\text { EGFR pos or } \\
\text { ALK pos }\end{array}$ \\
\hline
\end{tabular}

Abbreviations: GPA, graded prognostic assessment; KPS, Karnofsky Performance Status; ECM, extracranial metastases; BM, brain metastases; NA, not applicable; neg/unk, negative or unknown; NSCLC, non-small-cell lung cancer; pos, positive; EGFR, epidermal growth factor receptor; ALK, anaplastic lymphoma kinase. 
statistically significant. Hazard ratios (HRs) and 95\% confidence intervals (CIs) were calculated by Cox regression analyses.

\section{Results}

\section{Patient Characteristics}

During the period from February 2012 to June 2018, the records of 618 patients diagnosed with NSCLC and BM were enrolled in this retrospective study. A total of 524 patients were diagnosed with adenocarcinoma (ADC) and the remaining 94 patients were diagnosed with nonadenocarcinoma (non-ADC), which includes squamous cell lung cancer (42 patients), large cell lung cancer (20 patients) and cancers ( 32 patients). Because driver gene detection is not covered by government health insurance, only 352 (57.0\%) of patients' driver genes were determined, with 333 patients in the ADC group and 19 patients in the non-ADC. $E G F R$ or $A L K$-positive lesions were found in 224 (67.3\%) patients, with 218 patients in the ADC group and 6 patients in the non-ADC group. At the initial diagnosis, 240 patients presented with symptoms of BM, including 200 patients in the ADC group and 40 patients in the non-ADC group. The patient characteristics are detailed in Tables 2 and 3.

According to the Lung-molGPA score criteria detailed in Table 1, each patient in the group was given a score. As a result, $38.5 \%$ of patients $(238 / 618)$ and $45.3 \%$ of patients (280/618) scored 1.5-2 and 2.5-3, respectively, whereas only $7 \%$ (43/618) and $10.8 \%(67 / 618)$ scored $0-1$ and 3.5-4, respectively. In the ADC subgroup, 37 (7.1\%) patients scored 0-1, 201 (38.4\%) patients scored 1.5-2, 234 (44.6\%) patients scored $2.5-5$, and $52(9.9 \%)$ patients in score $3.5-4$ group, whereas was $6(6.4 \%)$ patients in score $0-1$ group, 37 (39.4\%) patients in score 1.5-2 group, 36 (38.3\%) patients in score 2.5-3 group and 15 (15.9\%) patients in score 3.5-4 group, in the non-ADC group, respectively (Table 4).

\section{Treatment Summary}

First-Line Systemic Treatment

Because the TKIs were not covered by the government health insurance before the year 2017 in China, only 118 patients (52.6\%) were given TKIs among the 224 patients harboring $\mathrm{EGFR}+$ or $\mathrm{ALK}+$, and all were first-generation including

Table 2 Univariate and Multivariate Cox Regression Analyses for OS and PFS in Adenocarcinoma Patients

\begin{tabular}{|c|c|c|c|c|c|c|}
\hline \multirow[t]{3}{*}{ Parameter } & \multirow[t]{3}{*}{ Categories } & \multirow[t]{3}{*}{$\mathbf{N}$} & \multicolumn{2}{|c|}{ Univariate } & \multicolumn{2}{|c|}{ Multivariate } \\
\hline & & & os & PFS & os & PFS \\
\hline & & & HR (95\% CI) & HR (95\% Cl) & HR $(95 \% \mathrm{CI})$ & HR (95\% Cl) \\
\hline \multirow[t]{3}{*}{ KPS } & $90-100$ & 210 & I & 1 & I & 1 \\
\hline & 80 & 263 & $1.59(1.07-2.36)$ & $1.17(0.91-1.49)$ & $1.35(0.89-2.04)$ & $1.10(0.71-1.69)$ \\
\hline & $<80$ & 51 & $1.34(0.69-2.62)$ & $1.04(0.68-1.58)$ & $1.19(0.60-2.35)$ & $1.16(0.76-1.77)$ \\
\hline \multirow[t]{2}{*}{ Age } & $<70$ & 459 & I & I & I & I \\
\hline & $\geq 70$ & 65 & $2.40(1.49-3.85)$ & $1.92(1.36-2.69)$ & $2.20(1.34-3.60)$ & $1.97(1.38-2.81)$ \\
\hline \multirow[t]{2}{*}{ ECM } & Absent & 154 & I & I & I & 1 \\
\hline & Present & 370 & $0.93(0.63-1.37)$ & $1.32(\mid .02-1.7 I)$ & I.06 (0.7I-I.59) & $1.45(1.10-1.90)$ \\
\hline \multirow[t]{3}{*}{ BM } & $1-4$ & 325 & I & I & I & 1 \\
\hline & $>4$ & 184 & I.5I (0.47-4.84) & $2.28(0.73-7.16)$ & I.43 (0.44-4.72) & $2.36(0.75-7.42)$ \\
\hline & NA & 15 & $0.98(0.67-1.42)$ & I.82 (0.58-5.74) & $0.97(0.66-1.42)$ & $2.03(0.64-6.45)$ \\
\hline \multirow[t]{3}{*}{ Gene Status } & EGFR pos or ALK pos & 218 & 1 & 1 & I & 1 \\
\hline & EGFR neg/unk and $A L K$ neg/unk & 115 & $2.31(1.44-3.70)$ & $1.47(1.10-1.98)$ & $2.17(1.33-3.54)$ & $1.49(1.10-2.02)$ \\
\hline & NA & 191 & $1.56(\mathrm{I} .0 \mathrm{I}-2.4 \mathrm{I})$ & $0.99(0.75-1.30)$ & $1.59(1.33-3.54)$ & $1.09(0.83-1.44)$ \\
\hline \multirow[t]{2}{*}{ Symptom } & No & 324 & I & I & I & 1 \\
\hline & Yes & 200 & $1.08(0.73-1.58)$ & $1.32(1.02-1.7 I)$ & $1.06(0.7 I-1.59)$ & $1.45(1.10-1.90)$ \\
\hline
\end{tabular}

Abbreviations: OS, overall survival; PFS, progression-free survival; KPS, Karnofsky Performance Status; ECM, extracranial metastases; BM, brain metastases. 
Table 3 Univariate and Multivariate Cox Regression Analyses for OS and PFS in Nonadenocarcinoma Patients

\begin{tabular}{|c|c|c|c|c|c|c|}
\hline \multirow[t]{3}{*}{ Parameter } & \multirow[t]{3}{*}{ Categories } & \multirow[t]{3}{*}{$\mathbf{N}$} & \multicolumn{2}{|c|}{ Univariate } & \multicolumn{2}{|c|}{ Multivariate } \\
\hline & & & os & PFS & os & PFS \\
\hline & & & HR (95\% Cl) & HR (95\% Cl) & HR (95\% Cl) & HR (95\% Cl) \\
\hline \multirow[t]{3}{*}{ KPS } & $90-100$ & 35 & 1 & I & 1 & 1 \\
\hline & 80 & 47 & I.79 (0.72-4.45) & $0.7 \mid(0.29-1.73)$ & $2.10(0.79-5.55)$ & $0.53(0.18-1.52)$ \\
\hline & $\leq 70$ & 12 & $2.57(0.88-7.49)$ & I.I4 (0.49-2.67) & $2.93(0.7 I-12.02)$ & $1.04(0.39-2.74)$ \\
\hline \multirow[t]{2}{*}{ Age } & $<70$ & 79 & $\mathrm{I}$ & I & I & 1 \\
\hline & $\geq 70$ & 15 & $0.76(0.23-2.54)$ & $1.30(0.55-3.06)$ & $0.68(0.19-2.49)$ & 1.51 (0.60-3.79) \\
\hline \multirow[t]{2}{*}{ ECM } & Absent & 45 & I & I & I & 1 \\
\hline & Present & 49 & $1.45(0.65-3.23)$ & $\mathrm{I} .03(0.59-\mathrm{I} .8 \mathrm{I})$ & $2.40(0.90-6.39)$ & $1.17(0.62-2.20)$ \\
\hline \multirow[t]{2}{*}{ BM } & $1-4$ & 66 & 1 & I & I & 1 \\
\hline & $>4$ & 28 & $2.61(1.20-5.67)$ & $1.08(0.60-1.98)$ & $2.60(0.96-7.01)$ & I.I $12(0.54-2.32)$ \\
\hline \multirow[t]{3}{*}{ Gene Status } & EGFR pos or ALK pos & 6 & I & I & I & I \\
\hline & EGFR neg/unk and $A L K$ neg/unk & 13 & $2.01(0.47-8.57)$ & $2.20(0.69-7.05)$ & $1.94(0.44-8.52)$ & $3.67(0.99-13.70)$ \\
\hline & NA & 75 & $0.60(0.05-6.59)$ & $1.21(0.43-3.44)$ & $0.11(0.01-1.75)$ & $1.75(0.56-5.50)$ \\
\hline \multirow[t]{2}{*}{ Symptom } & No & 54 & 1 & I & 1 & 1 \\
\hline & Yes & 40 & $1.12(0.52-2.46)$ & $1.07(0.60-1.89)$ & $1.84(0.73-4.63)$ & $0.89(0.46-\mid .7 I)$ \\
\hline
\end{tabular}

Abbreviations: OS, overall survival; PFS, progression-free survival; KPS, Karnofsky Performance Status; ECM, extracranial metastases; BM, brain metastases.

Table 4 Comparison Survival in Patients with NSCLC

\begin{tabular}{|l|c|c|c|c|}
\hline \multirow{2}{*}{$\begin{array}{l}\text { Score } \\
\text { Sung GPA }\end{array}$} & \multicolumn{2}{|c|}{$\begin{array}{c}\text { Adenocarcinoma } \\
\text { NSCLC Lung- } \\
\text { molGPA }\end{array}$} & \multicolumn{2}{|c|}{$\begin{array}{c}\text { Nonadenocarcinoma } \\
\text { NSCLC Lung- } \\
\text { molGPA }\end{array}$} \\
\cline { 2 - 5 } & $\begin{array}{c}\text { MS, } \\
\text { Mo }\end{array}$ & $\begin{array}{c}\text { Patients, } \\
\text { No. (\%) }\end{array}$ & $\begin{array}{c}\text { MS, } \\
\text { Mo }\end{array}$ & $\begin{array}{c}\text { Patients, No. } \\
\text { (\%) }\end{array}$ \\
\hline $0-1$ & 25 & $37(7.06 \%)$ & 12 & $6(6.38 \%)$ \\
\hline $1.5-2$ & 30 & $201(38.36 \%)$ & 20.2 & $37(39.36 \%)$ \\
\hline $2.5-3$ & 35 & $234(44.66 \%)$ & 29 & $36(38.30 \%)$ \\
\hline $3.5-4$ & NA & $52(9.92 \%)$ & 33 & $15(15.96 \%)$ \\
\hline Overall & 33.6 & $524(100 \%)$ & 28 & $94(100 \%)$ \\
\hline
\end{tabular}

Abbreviations: GPA, graded prognostic assessment; MS, median survival; NSCLC, non-small-cell lung cancer.

gefitinib, erlotinib, and icotinib. At the last follow-up, 97 patients had experienced disease progression, and the second biopsy was conducted in 75 patients (77.3\%). As a result, 40 patients (53.3\%) harbored T790M mutation, and of these, 32 patients received the third-generation TKI, osimertinib.

The remaining 500 patients were given platinum-based chemotherapy as the first-line treatment, which included pemetrexed, paclitaxel, and gemcitabine. In the 106 patients harboring $E G F R+, 80$ patients were given TKI as the second-line treatment when the patients experienced suffered from disease progression.

\section{Local Therapy to Brain Metastasis}

The local treatment for the 391 patients with 1-4 BM was as follows: WBRT \pm boost (or simultaneous integrated boost) to 135 patients (34.5\%); SRS (or SBRT) alone to 202 patients (51.7\%); and surgery alone to 54 patients (13.8\%).

For 212 patients with more than 4 BM, 182 patients (85.8\%) received WBRT, while 30 patients (14.2\%) received SBRT alone. For the 15 patients with not applicable information, WBRT was conducted in 12 patients and WBRT+SRS in 3 patients.

\section{Overall Survival}

The last follow-up was 31 August, 2019. The median follow-up time of the whole group was 26.8 months. The median survival time (MST) of the whole group and the OS rate at 3 years were 33.0 months and $44.5 \%$, respectively. For patients with ADC, the MST and the OS rates at 
Non-adenocarcinoma

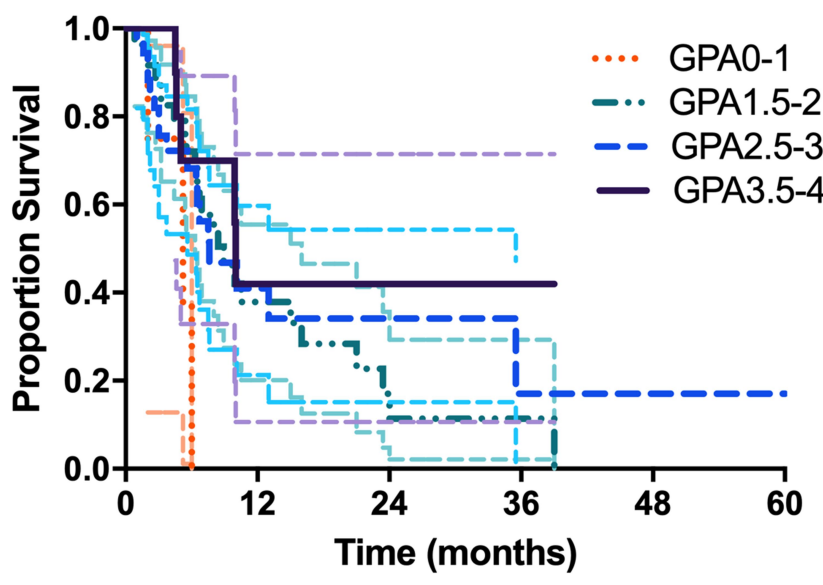

Figure I Survival curves of patients with ADC and non-ADC.

3 years were 33.6 months and $45.6 \%$, which were higher than the 28 months and $37.2 \%$ observed in the subgroup with non-ADC but the difference was not significant $(p=0.21)$. The survival curves are compared in Figure 1 and in Table 4.

\section{Impacts of Clinical Characteristics on OS and PFS}

For ADC patients, on univariate analysis, older age, lower KPS, presence of ECM, additional brain metastatic lesions, EGFR negative/unknown, and $A L K$ negative/ unknown were significantly associated with increased risk of death and disease progression of ADC patients with $\mathrm{BM}(\mathrm{HR}>1)$. As we can see in Table 2, multivariate analysis demonstrated that older age, worse performance status, presence of ECM, and local brain treatment were significantly associated with an increased risk of death and disease progression of ADC patients with $\mathrm{BM}(\mathrm{HR}>1)$. Furthermore, univariate and multivariate Cox regression analyses showed the same results in terms of prognostic factors for non-ADC patients.

\section{Survival Stratified by Lung-molGPA for NSCLC}

For ADC patients, the survival rates of patients stratified according to the four prognostic classes are detailed in Table 3. For 52 patients with Lung-molGPA scores of 3.5 to 4.0 , the MST was not reached. However, this group had a median survival of more than 4 years. The survival difference between the four score classes was significant $(p=0.048)$ (Figure 2). For non-ADC patients,

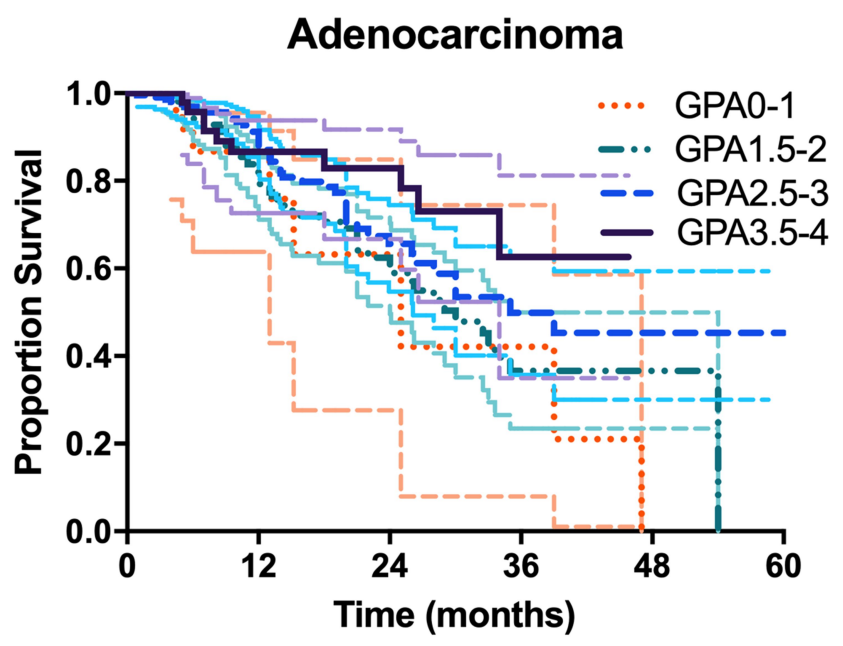

Figure 2 Survival curves stratifying lung adenocarcinoma patients by the LungmolGPA.

only 15 patients had Lung-molGPA scores of 3.5 to 4.0 (n = 15), the MST in this subgroup was 33 months. The survival difference between the four score classes was significant ( $p=0.017$ ) (Figure 3).

For ADC patients, there was no statistically significant difference across groups stratified by the Lung-molGPA scores $(p=0.437$ for $0-1$ vs $1.5-2 ; p=0.072$ for $1.5-2$ vs 2.5-3; and $p=0.304$ for $2.5-3$ vs 3.5-4). However, the MST in the score 1.5-2 group was significantly lower than that in the score 3.5-4 group (30 months vs not reached, $p=0.049$ ).

Similarly, no statistically significant difference in survival was observed for groups stratified by the LungmolGPA in non-ADC patients $(p=0.08$ for $0-1$ vs $1.5-2 ; p=0.26$ for $1.5-2$ vs $2.5-3 ; p=0.38$ for $2.5-3$ vs

\section{Non-adenocarcinoma}

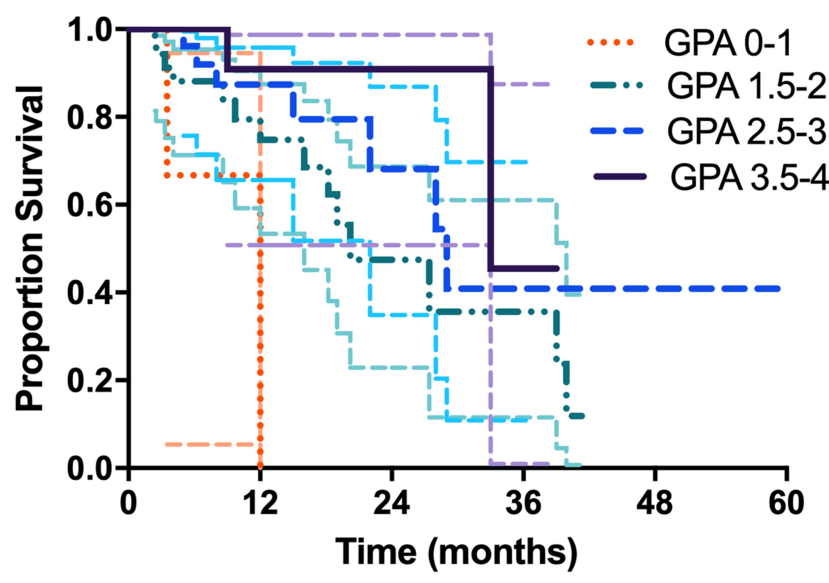

Figure 3 Survival curves stratifying lung non-adenocarcinoma patients by the LungmolGPA. 
3.5-4). However, the survival in patients scoring 0-1 was significantly lower than that in patients scoring 2.5-3 or $3.5-4$ ( $p=0.02$ and $p=0.005$, respectively).

PFS in ADC patients scoring 3.5-4 was significantly longer than in patients with scores of $0-1$ or $1.5-2$ (17 months vs 9 months, $p=0.01 ; 17$ months vs 10 months, $p=0.022$; respectively; Figure S1). In addition, the PFS with score $0-1$ was significantly lower than that with score $1.5-2(p=0.041$; Figure S2). As we can see in Table S1, we found that the baseline clinical features were matched between the two groups, however, more EGFR/ALKpositive patients in adenocarcinoma group compared with nonadenocarcinoma group. These suggest that adenocarcinoma patients have more treatment options such as TKIs, so adenocarcinoma patients have a longer survival and better prognosis.

\section{Discussion}

$\mathrm{BM}$ are a serious risk factor for poor survival in patients with NSCLC. Emerging evidence has revealed that patients with BM could benefit from targeted therapy and immunotherapy. 8 Therefore, we identified patient subgroups stratified by survival outcomes based on the patient's intrinsic characteristics that were associated with the combination of treatment strategies. Patients wild type for mutations aside from radiotherapy underwent treatment with mono-chemotherapy according to guidelines for elderly people. ${ }^{27}$ RPA, GPA, and DS-GPA have been validated to predict survival, ${ }^{10,11}$ but they no longer meet the clinical needs given the availability of new treatment strategies. Sperduto et al proposed a Lung-molGPA model, which was based on age, KPS, extracranial metastases, number of BM, EGFR and ALK alterations, to stratify patients with different survival outcomes. ${ }^{7}$ The final results showed the median survival for ADC patients with Lung-molGPA scores of $0-1,1.5-2,2.5-3$, and 3.5-4 were 6.9 months, 13.7 months, 26.5 months, and 46.8 months, respectively ( $p=0.03$ for $0-1$ vs $1.5-2 ; p<0.001$ for $1.5-2$ vs $2.5-3$; and $p<0.001$ for $2.5-3$ vs 3.5-4.0). Similarly, the survival of non-ADC patients with lung GPA scores $0-1,1.5-2$, and 2.5-3 were 5.3 months, 9.8 months, and 12.8 months, respectively ( $p<0.001$ for $0-1$ vs $1.5-2$; and $p=0.04$ for $1.5-2$ vs $2.5-3)$. However, no studies focusing on the LungmolGPA model have been performed to validate their clinical utility since then.

In the present study, we retrospectively reviewed 618 Asian patients newly diagnosed NSCLC with BM to evaluate the utility of the Lung-molGPA model. The MST in the ADC group was prolonged as the Lung-molGPA score increased; however, there was no significant difference in each of the groups. Notably, the MST for patients with score 3.5-4 was more than 4 years, which was significantly longer than that for patients with score 1.5-2 (30 months, $p=0.049$ ). Moreover, PFS in ADC patients with a score of 3.5-4 was significantly longer than patients having scores $0-1$ or 1.5-2 (17 months vs 9 months, $p=$ $0.01 ; 17$ months vs 10 months, $p=0.022$; respectively; Figure $\mathrm{S} 1 \mathrm{new})$. This study was the first to consider the effects of driver genes in non-ADC and to investigate its impact on stratification of patient outcomes. Nineteen patients (19/94, 20.2\%) underwent genetic testing of driver genes, of whom 6 patients $(31.5 \%)$ were identified with $E G F R$ or $A L K$ mutations. OS with scores $0-1,1.5-2$, 2.5-3, and 3.5-4 according to the Lung-molGPA grade was 12 months, 20.2 months, 29 months, and 33 months, in the non-ADC group, respectively, and OS was not significantly different across groups. Nonetheless, the worst survival was observed in patients who scored $0-1$, which was significantly lower than patients who scored $2.5-3$ and 3.5-4 ( $p=0.02$ and $p=0.005$, respectively). In addition, the PFS observed in patients with a score of $0-1$ was significantly lower than in those with scores of $1.5-2$ ( $p=0.041$; Figure S2 new).

The Lung-molGPA, as a new prognostic tool, provides an accurate estimation of prognosis for NSCLC patients with BM. ${ }^{7}$ Patient age, KPS, presence of extracranial metastases, number of $\mathrm{BM}$, and positive findings for EGFR and ALK were added to the Lung-molGPA. ${ }^{12,13}$ In this study, consistent with previously published data, ${ }^{14-16}$ age, Karnofsky Performance Status, extracranial metastases, number of $\mathrm{BM}$, and gene status, were all risk factors for OS in both ADC and non-ADC patients. ${ }^{28}$ The LungmolGPA was verified in NSCLC patients with BM. Although there was no statistically significant difference between the four groups, there were significant differences in median survival and survival rates. In the present study, NSCLC patients with BM showed a median OS of 11 months, which was slightly longer than the median OS of 8.6 months reported in Hendriks et al's study. ${ }^{17}$ This survival advantage might be associated with the widespread use of molecularly-targeted agents as a treatment strategy. Moreover, additional treatment options after disease progression may have also contributed to improved survival.

An excellent clinical tool should have the ability to guide the choice of clinical treatment strategies. A secondary analysis of RTOG 95-08 indicated 
a survival advantage regardless of the patient had 1,2 , or 3 BM for patients with a high GPA (3.5-4). ${ }^{18}$ This result strengthens the observations that SRS, when given in association with WBRT, improves local disease control and OS in those patients with optimal prognostic factors. Another retrospective study, including 164 patients treated with SRS alone or SRS plus WBRT, indicated that the MST stratified by DS-GPA was consistent with the OS associated with BM in NSCLC DS-GPA and did not correlate with SRS plus WBRT or SRS alone. ${ }^{19}$ According to the current evidence, only patients with specific characteristics (such as no more than $4 \mathrm{BM}$ ) should be considered for SRS. ${ }^{20,21}$ Nagtegaal et al conducted a retrospective study to assess the feasibility of DSGPA in 367 NSCLC patients treated with SRS for BM. ${ }^{22}$ Their findings indicated that the median survival was significantly shorter in the ADC NSCLC subgroup with GPA scores of 2.5-3 (15.4 months vs 26.5 months), but in other groups, it was largely comparable to the corresponding DS-GPA cohorts. The difference may be due to the specific characteristics of patients treated with SRS versus other patients. Hence, the value of DS-GPA as a useful prognostic tool was confirmed for counseling of individual patients with BM before undergoing SRS. Furthermore, for patients treated with WBRT, the MST for GPA 0-1 with elevated serum lactate dehydrogenase (LDH) was only 37 days compared to 123 days in patients without elevated LDH $(p<0.001) .{ }^{23}$ Hence, the stratification of prognosis according to GPA before treatment is necessary and contributes to the choice of treatment. Conversely, targeted therapy as the first-line treatment for patients with $E G F R$ mutations with $\mathrm{BM}$ is now widely recognized. In addition, earlier guidelines recommended treatment with EGFR TKIs was suitable for locally advanced or metastatic NSCLC after the failure of the first-line chemotherapy. ${ }^{24,25}$

In summary, if the patient presents with 1 to $3 \mathrm{BM}$ with a high-grade GPA (3.5-4), SRS can be given combined with WBRT; for patients with EGFR mutations and BM, targeted therapy is an optional strategy with or without radiation. However, if patients with a low Lung-molGPA $(0-1),{ }^{26}$ best supportive care is probably the better treatment.

It is crucial to derive benefit from an aggressive treatment approach for patients with BM. While validating this model, we found that OS and PFS of ADC patients with symptomatic BM were significantly affected. The MST in patients scoring 2.5-3 and 1.5-2 with asymptomatic BM was 28 months and 21 months, respectively, while OS was 39 months and 32.5 months in patients with symptomatic BM. Hence, the treatment of patients with asymptomatic BM may be overlooked. The predictive ability of LungmolGPA can be considered an estimate of the expected survival on an individual level, which aids the physician in providing an accurate estimation of prognosis to the patient. Meanwhile, it can also be used as a tool to help determine the most suitable treatment and intensity of follow-up.

However, some limitations are inevitable as this was a retrospective study. First, the number of patients with non-ADC was small. Second, few patients underwent genetic testing. Nevertheless, the survival between scores $0-1$ and $2.5-3 / 3.5-4$ was statistically significant, which is sufficient to support the feasibility of the clinical application of the Lung-molGPA score. Third, all patients used in this study were from a single institution; thus, the data presented herein might not reflect the reality of other centers. A larger population from multiple institution is needed to balance the variations among various hospitals to further validate the value of Lung-molGPA.

\section{Conclusion}

In conclusion, the Lung-molGPA was verified in our retrospective study and was demonstrated to be a useful clinical tool in clinical decision making regarding patient treatment and predict prognosis. However, with the development of novel checkpoint inhibitors and related predictive indicators, static models are no longer suitable. Hence, this model is still exploratory and needs further external validation. In the future, combining more potential indicators, such as PD-L1 expression and markers related to immunity, will improve the model and further guide treatment options to better predict prognosis. More samples are needed to detect and verify the effects of BM symptoms in NSCLC patients.

\section{Ethics Approval}

This study was approved by the ethical review board of Shandong Cancer Hospital and Institute and complied with the Helsinki declaration. Written informed consent was obtained from all participants.

\section{Author Contributions}

Ji Li and Wang Jing made a significant contribution to the work reported, whether that is in the conception, study design, execution, acquisition of data, analysis and interpretation, or in all these areas. Xiaoyang Zhai and 
Wenxiao Jia have drafted or written, or substantially revised or critically reviewed the article. Hui Zhu have agreed on the journal to which the article will be submitted. Hui Zhu and Jinming Yu reviewed and agreed on all versions of the article before submission, during revision, the final version accepted for publication, and any significant changes introduced at the proofing stage. Jinming Yu agree to take responsibility and be accountable for the contents of the article. All authors contributed to data analysis, drafting or revising the article, have agreed on the journal to which the article will be submitted, gave final approval of the version to be published, and agree to be accountable for all aspects of the work.

\section{Funding}

This research was funded by Innovation Project of Shandong Academy of Medical Science to Jinming Yu, National Natural Science Foundation of China (Grant number 81972862), and Wu Jieping Medical Foundation (Grant number 320675018288).

\section{Disclosure}

The authors report no conflicts of interest in this work.

\section{References}

1. Chen W, Zheng R, Baade PD, et al. Cancer statistics in China, 2015. CA Cancer J Clin. 2016;66(2):115-132. doi:10.3322/caac.21338

2. Torre LA, Bray F, Siegel RL, et al. Global cancer statistics, 2012. $C A$ Cancer J Clin. 2015;65(2):87-108. doi:10.3322/caac.21262

3. Andre F, Grunenwald D, Pujol JL, et al. Patterns of relapse of N2 nonsmall-cell lung carcinoma patients treated with preoperative chemotherapy: should prophylactic cranial irradiation be reconsidered? Cancer. 2001;91(12):2394-2400. doi:10.1002/1097-0142(20010615) 91:12<2394::AID-CNCR1273>3.0.CO;2-6

4. Kumar P, Herndon J, Langer M, et al. Patterns of disease failure after trimodality therapy of nonsmall cell lung carcinoma pathologic stage IIIA (N2): analysis of cancer and leukemia group B protocol 8935. Cancer. 1996;77(11):2393-2399. doi:10.1002/(SICI)1097-0142(1996 0601)77:11<2393::AID-CNCR31>3.0.CO;2-Q

5. Gaspar L, Scott C, Rotman M, et al. Recursive Partitioning Analysis (RPA) of prognostic factors in three Radiation Therapy Oncology Group (RTOG) brain metastases trials. Int J Radiat Oncol Biol Phys. 1997;37(4):745-751. doi:10.1016/S0360-3016(96)00619-0

6. Sperduto PW, Kased N, Roberge D, et al. Summary report on the graded prognostic assessment: an accurate and facile diagnosis-specific tool to estimate survival for patients with brain metastases. J Clin Oncol. 2012;30(4):419-425. doi:10.1200/JCO.20 11.38 .0527

7. Sperduto PW, Yang TJ, Beal K, et al. Estimating survival in patients with lung cancer and brain metastases an update of the graded prognostic assessment for lung cancer using molecular markers (Lung-molGPA). JAMA Oncol. 2017;3(6):827-831. doi:10.1001/ jamaoncol.2016.3834

8. Wang Z, Yang JJ, Tu HY, et al. Retrospective study on bevacizumab in the treatment of non-small cell lung cancer with brain metastases. Int J Clin Oncol. 2019. doi:10.1007/s10147-019-01552-5
9. Zhuang H, Shi S, Chang JY. Treatment modes for EGFR mutations in patients with brain metastases from non-small cell lung cancer: controversy, causes, and solutions. Transl Lung Cancer Res. 2019;8 (4):524-531. doi:10.21037/tlcr.2019.07.03

10. Sperduto PW, Berkey B, Gaspar LE, et al. A new prognostic index and comparison to three other indices for patients with brain metastases: an analysis of 1960 patients in the RTOG database. Int J Radiat Oncol Biol Phys. 2008;70(2):510-514. doi:10.1016/j.ijrobp.2007. 06.074

11. Liu S, Chen P, Liu YW, et al. Role of recursive partitioning analysis and graded prognostic assessment on identifying non-small cell lung cancer patients with brain metastases who may benefit from postradiation systemic therapy. Chin Med $J$ (Engl). 2018;131(10): 1206-1213. doi:10.4103/0366-6999.231517

12. Sperduto PW, Yang TJ, Beal K, et al. The effect of gene alterations and tyrosine kinase inhibition on survival and cause of death in patients with adenocarcinoma of the lung and brain metastases. Int J Radiat Oncol Biol Phys. 2016;96(2):406-413. doi:10.1016/j. ijrobp.2016.06.006

13. Yamamoto M, Serizawa T, Shuto T, et al. Stereotactic radiosurgery for patients with multiple brain metastases (JLGK0901): a multi-institutional prospective observational study. Lancet Oncol. 2014;15(4):387-395. doi:10.1016/S1470-2045(14)70061-0

14. Wu C, Zhao C, Yang Y, et al. High discrepancy of driver mutations in patients with NSCLC and synchronous multiple lung ground-glass nodules. J Thorac Oncol. 2015;10(5):778-783. doi:10.1097/JTO.000 0000000000487

15. Kancha RK, Von Bubnoff N, Peschel C, et al. Functional analysis of epidermal growth factor receptor (EGFR) mutations and potential implications for EGFR targeted therapy. Clin Cancer Res. 2009;15 (2):460-467. doi:10.1158/1078-0432.CCR-08-1757

16. Chen K, Yu X, Zhang F, et al. Applicability of the lung-molGPA index in non-small cell lung cancer patients with different gene alterations and brain metastases. Lung Cancer. 2018;125:8-13. doi:10.1016/j.lungcan.2018.08.023

17. Hendriks LEL, Henon C, Auclin E, et al. Outcome of patients with non-small cell lung cancer and brain metastases treated with checkpoint inhibitors. J Thorac Oncol. 2019;14(7):1244-1254. doi:10. 1016/j.jtho.2019.02.009

18. Sperduto PW, Shanley R, Luo X, et al. Secondary analysis of RTOG 9508, a Phase 3 randomized trial of whole-brain radiation therapy versus WBRT plus stereotactic radiosurgery in patients with 1-3 brain metastases; poststratified by the graded prognostic assessment (GPA). Int J Radiat Oncol Biol Phys. 2014;90(3):526-531. doi:10.10 16/j.jijrobp.2014.07.002

19. Woody NM, Greer MD, Reddy CA, et al. Validation of the disease-specific GPA for patients with 1 to 3 synchronous brain metastases in newly diagnosed NSCLC. Clin Lung Cancer. 2018;19 (1):e141-e147. doi:10.1016/j.cllc.2017.06.011

20. Hartgerink D, van der Heijden B, De Ruysscher D, et al. Stereotactic radiosurgery in the management of patients with brain metastases of non-small cell lung cancer: indications, decision tools and future directions. Front Oncol. 2018;8:154. doi:10. 3389/fonc. 2018.00154

21. Chao ST, De Salles A, Hayashi M, et al. Stereotactic radiosurgery in the management of limited (1-4) brain metasteses: systematic review and international stereotactic radiosurgery society practice guideline. Clin Neurosurg. 2018;83(3):345-353. doi:10.1093/neuros/nyx522

22. Nagtegaal S, Claes A, Suijkerbuijk K, et al. Comparing survival predicted by the diagnosis-specific Graded Prognostic Assessment (DS-GPA) to actual survival in patients with 1-10 brain metastases treated with stereotactic radiosurgery. Radiother Oncol. 2019;13 8:173-179. doi:10.1016/j.radonc.2019.06.033

23. Miyazawa K, Shikama N, Okazaki S, et al. Predicting prognosis of short survival time after palliative whole-brain radiotherapy. $J$ Radiat Res. 2018;59(1):43-49. doi:10.1093/jrr/rrx058 
24. Kim JE, Lee DH, Choi Y, et al. Epidermal growth factor receptor tyrosine kinase inhibitors as a first-line therapy for never-smokers with adenocarcinoma of the lung having asymptomatic synchronous brain metastasis. Lung Cancer. 2009;65(3):351-354. doi:10.1016/j. lungcan.2008.12.011

25. Wu YL, Zhou C, Cheng Y, et al. Erlotinib as second-line treatment in patients with advanced non-small-cell lung cancer and asymptomatic brain metastases: a Phase II study (CTONG-0803). Ann Oncol. 2013;24(4):993-999. doi:10.1093/annonc/mds529

26. Chen K, Zhang F, Fan Y, et al. Lung-molGPA index predicts survival outcomes of non-small-cell lung cancer patients with synchronous or metachronous brain metastases. Onco Targets Ther. 2020;13:88 37-8844. doi:10.2147/OTT.S255478
27. Camerini A, Banna GL, Cinieri S, et al. Metronomic oral vinorelbine for the treatment of advanced non-small cell lung cancer: a multicenter international retrospective analysis. Clin Transl Oncol. 2019;21(6):790-795. doi:10.1007/s12094-018-1989-y

28. Sini C, Tuzi A, Rossi G, et al. Acquired resistance in oncogene-addicted non-small-cell lung cancer. Future Oncol. 2018; 14(13s):29-40. doi:10.2217/fon-2018-0097

\section{Publish your work in this journal}

OncoTargets and Therapy is an international, peer-reviewed, open access journal focusing on the pathological basis of all cancers, potential targets for therapy and treatment protocols employed to improve the management of cancer patients. The journal also focuses on the impact of management programs and new therapeutic

Submit your manuscript here: https://www.dovepress.com/oncotargets-and-therapy-journal agents and protocols on patient perspectives such as quality of life, adherence and satisfaction. The manuscript management system is completely online and includes a very quick and fair peer-review system, which is all easy to use. Visit http://www.dovepress.com/ testimonials.php to read real quotes from published authors. 